\title{
The matrix model of vortex-beam quadrefringence in a uniaxial crystal
}

\author{
Fadeyeva T.A., Rubass A.F. and Volyar A.V. \\ Physics Department, Taurida National V. Vernadsky University, \\ 4 Vernadsky Ave., 95007 Simferopol, Ukraine, E-mail: volyar@crimea.edu
}

Received: 28.03.2009

\begin{abstract}
We consider a matrix model for the tilted oblique propagation of singular beams in optically uniaxial crystals. The model predicts a series of fine effects such as vortex conversion and beam quadrefringence, being in a good agreement with the paraxial theory. Although the model gives overrated magnitudes of the beam parameters in the asymptotic case it enables us to calculate the processes occurring with high-order vortex beams, where the theory encounters computation difficulties.
\end{abstract}

Keywords: optical vortex, quadrefringence, uniaxial crystal

PACS: 41.85.-p, 42.25.Ja, 42.25.Lc

UDC: 535.5

\section{Introduction}

Among a variety of vortex transformations in singular beams [1] travelling through optically uniaxial crystals [2,3], a series of effects have drawn an unbiased attention because of their unexpected features. These are double-vortex generation in a fundamental Gaussian beam propagating in crystals along their optic axis $[4,5]$ and a vortex-beam quadrefringence in a tilted vortex-beam [6,7]. The latter effect represents splitting of initial tilted vortex-beam into four ones: the first two beams are a result of ordinary crystal birefringence and the second two ones are moving off the first ones via a spin-orbit coupling in the crystal [8]. However, transverse shift of the beams is very small (about a wavelength) [7] and so the effect has been perceived experimentally as an ordinary birefringence of the vortex-beam. The beam transformations have been described in the framework of beam solutions of the paraxial wave equations [9]. The theoretical expressions for the beam amplitudes have been presented in a very cumbrous mathematic form so that their analytical analysis meets serious difficulties, thus provoking the corresponding computer simulations as the only reliable way out.

On the other hand, in order to simplify the computation process, a series of models of the effect has been proposed $[4,10,11,12]$. The model presented in the works $[4,10]$ has been based on a matrix representation of symmetric bundle of rays in anisotropic crystals, including gyrotropic ones. This comprehend correctly the beam propagation along the crystal optic axis and the generation of double-charged vortex but could not embrace the case of propagation of tilted beams, when the bundle of rays gets 
asymmetric. The model suggested by the authors [11, 12] involves two similar tilted vortex-beams with orthogonal linear polarizations, propagating at different angles to the optic axis. This model has allowed describing fine polarisation structure of the vortexbeam in the intermediate range of inclination angles, beginning from the on-axis propagation (excluding the beam propagation along the optic axis) up to the angle of beam splitting (a so-called undistinguished border [13]). However, it does not take into account a degenerate case of the on-axis beam propagation and the vortex-generation and cannot predict the asymptotic transverse shift of the beams. As a result, the model [11, 12] has proven to be in conflict with the conservation law for the angular momentum flux in crystal $[7,14]$.

The new matrix model presented in this paper enables us to describe both the degenerate case and the asymptotic beam splitting in the crystal. It gives also adequate description of fine polarisation structure of the beam in the intermediate range of the inclination angles.

\section{The matrix model}

At first we assume that a symmetric light beam propagates along the optic axis of an unbounded homogeneous, optically uniaxial crystal with a permittivity tensor in its diagonal form: $\operatorname{diag} \hat{\varepsilon}=\operatorname{diag}\left(\varepsilon_{o}, \varepsilon_{o}, \varepsilon_{3}\right)$, where $n_{o}=\sqrt{\varepsilon_{o}}$ and $n_{3}=\sqrt{\varepsilon_{3}}$ are the refractive indices along the principal crystallographic axes and $n_{o}>n_{3}$. Some ray of this beam propagates obliquely at the angle $\vartheta$ with respect to the crystal optic axis (see Fig. 1) and intersects the plane $z=$ const at the point $\{r, \varphi\}$. Transformation of the complex amplitude $\boldsymbol{\Psi}_{r a y}=\left(\begin{array}{l}\Psi_{+} \\ \Psi_{-}\end{array}\right)$of the ray in a circularly polarised basis may be defined as [4, 15]

$$
\left(\begin{array}{l}
\Psi_{+} \\
\Psi_{-}
\end{array}\right)_{z}=\left(\begin{array}{cc}
\cos \delta & e^{-i 2 \varphi} \\
e^{i 2 \varphi} & \cos \delta
\end{array}\right)\left(\begin{array}{l}
\Psi_{+} \\
\Psi_{-}
\end{array}\right)_{0},
$$
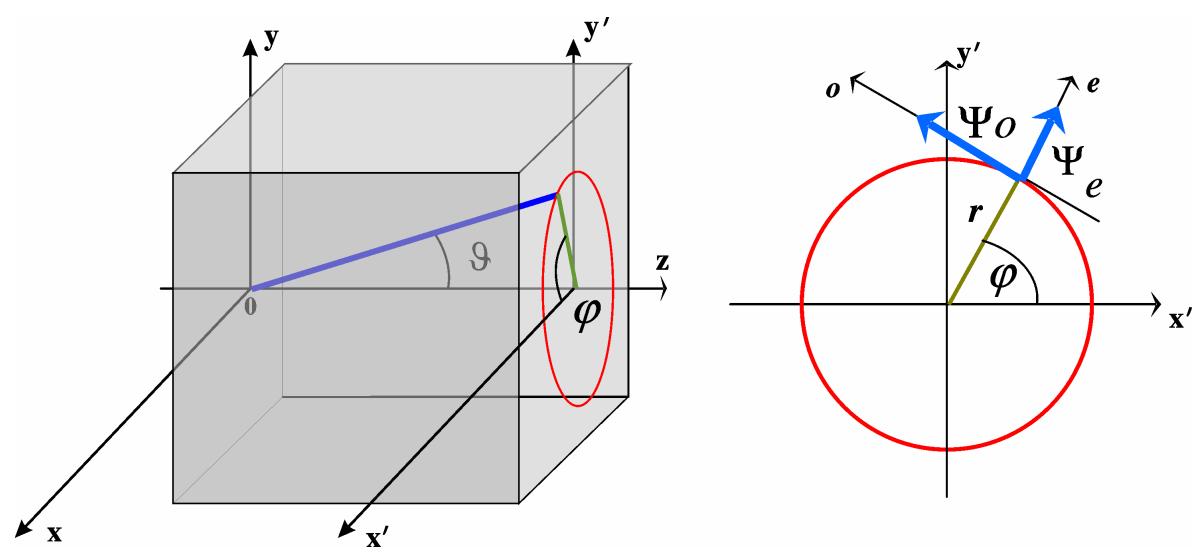

Fig. 1. Sketched representation of the beam propagation through crystal.

Optic axis of the crystal is parallel to $z$ axis. 
where $\delta=\frac{1}{2} \Delta n k R \sin ^{2} \vartheta=\frac{1}{2} \Delta n k \frac{r^{2}}{R}$ stands for the phase difference between the ordinary and extraordinary rays [16], $\Delta n=n_{o}-n_{3}$ and $R^{2}=r^{2}+z^{2}, r^{2}=x^{2}+y^{2}$. Assuming that the beam consists of rays with the same amplitudes and the wave vectors $\mathbf{k}_{i}$ directed along a radius $R$ of a semisphere, one can generalise Eq. (1) to the case of spherical wave with the amplitude $\mathbf{E}_{s p h}=\boldsymbol{\Psi} \frac{e^{-i k R}}{R}$. Note that the polarisation distribution at the plane $\mathrm{z}=0$ (see Fig. 1) given by the above relation remains valid only for a narrow range of angles $\vartheta\left(\sin \vartheta \approx \vartheta, \cos \vartheta \approx 1-\vartheta^{2} / 2\right)$.

Our model representation is as follows. The transfer from the spherical wave to the paraxial Gaussian beam is modelled by the substitution $z \rightarrow z+i z_{j}$ (with $z_{j}$ being a real constant and $j=o, e$ ) [17] and the substitutions

$$
R \rightarrow R_{j}=\sqrt{\left(z+i z_{j}\right)^{2}+r^{2}}=\left(z+i z_{j}\right) \sqrt{1+\frac{r^{2}}{\left(z+i z_{j}\right)^{2}}} \simeq\left(z+i z_{j}\right)+\frac{r^{2}}{2\left(z+i z_{j}\right)}
$$

in the numerator of the exponent $e^{-i k R}$ and $R \rightarrow R_{j} \rightarrow z+i z_{j}$ in the denominator of Eq. (1). To trace a proper order of those substitutions, we rewrite Eq. (1) as

$$
\begin{aligned}
& \boldsymbol{\Psi}_{r a y} \rightarrow \boldsymbol{\Psi}(z)=\frac{1}{2}\left[\left(\begin{array}{cc}
1 & e^{-i 2 \varphi} \\
e^{i 2 \varphi} & 1
\end{array}\right) e^{i \delta}\left(\begin{array}{l}
1 \\
0
\end{array}\right)+\left(\begin{array}{cc}
1 & -e^{-i 2 \varphi} \\
-e^{i 2 \varphi} & 1
\end{array}\right) e^{-i \delta}\left(\begin{array}{l}
1 \\
0
\end{array}\right)\right] \Psi_{0}= \\
& =\frac{1}{2}\left(\begin{array}{cc}
\Psi_{0} e^{i \delta}+\Psi_{0} e^{-i \delta} & e^{-i 2 \varphi}\left(\Psi_{0} e^{i \delta}-\Psi_{0} e^{-i \delta}\right) \\
e^{i 2 \varphi}\left(\Psi_{0} e^{i \delta}-\Psi_{0} e^{-i \delta}\right) & 1
\end{array}\right)\left(\begin{array}{l}
1 \\
0
\end{array}\right) \rightarrow \frac{1}{2}\left(\begin{array}{c}
\Psi_{o}+\Psi_{e} \\
e^{i 2 \varphi}\left(\Psi_{o}-\Psi_{e}\right)
\end{array}\right)
\end{aligned}
$$

where $\Psi_{0}$ is the complex amplitude of the initial beam. Then we modify the beam functions in the following way:

$$
\begin{aligned}
\Psi_{o} & =\frac{e^{-i k z}}{z+i z_{o}} \exp \left(-i \frac{k r^{2}}{2\left(z+i z_{o}\right)}+i \delta\right) \\
& =\frac{e^{-i k z}}{z+i z_{o}} e \operatorname{xp}\left(-i \frac{\left(k+k_{0} \Delta n\right) r^{2}}{2\left(z+i z_{o}\right)}\right) \rightarrow \frac{z_{o} e^{-i k_{o} z}}{z+i z_{o}} \exp \left(-i \frac{k_{o} r^{2}}{2\left(z+i z_{o}\right)}\right), \\
\Psi_{e} & =\frac{e^{-i k z}}{z+i z_{e}} e \operatorname{xp}\left(-i \frac{k r^{2}}{2\left(z+i z_{e}\right)}-i \delta\right) \\
& =\frac{e^{-i k z}}{z+i z_{e}} e \operatorname{xp}\left(-i \frac{\left(k-k_{0} \Delta n\right) r^{2}}{2\left(z+i z_{e}\right)}\right) \rightarrow \frac{z_{o} e^{-i k_{o} z}}{z+i z_{o}} e \operatorname{xp}\left(-i \frac{k_{e} r^{2}}{2\left(z+i z_{e}\right)}\right) .
\end{aligned}
$$

This is a trait of our model. The ordinary $\left(\Psi_{o}\right)$ and extraordinary $\left(\Psi_{e}\right)$ beams 
propagate along the optic axis with the same wavenumbers $\left(k_{o}\right)$, though their complex amplitudes $\Psi_{o}\left(r, z, k_{o}\right)$ and $\Psi_{e}\left(r, z, k_{e}\right)$ depend on different wavenumbers ( $k_{o}$ and $k_{e}$, respectively). The modification itself is valid for relatively small crystal lengths and can result in unpredictable deviations for the large distances. We cannot define the values $k_{o}$ and $k_{e}$ in framework of the given model and so use their magnitudes taken from the paraxial theory of beams [9]: $k_{o}=k_{0} n_{o}, k_{e}=k_{0} \frac{n_{3}^{2}}{n_{o}}$, with $k_{0}$ being the wavenumber for the free space. Thus, the complex amplitudes of the beams in our model are written as

$$
\Psi_{o}=\frac{1}{\sigma_{o}} e \operatorname{xp}\left(-i \frac{r^{2}}{w_{0}^{2} \sigma_{o}}\right), \quad \Psi_{e}=\frac{1}{\sigma_{o}} \exp \left(-\frac{r^{2}}{w_{0}^{2} \sigma_{e}}\right),
$$

where $\sigma_{o, e}=1-i \frac{z}{z_{o, e}}, z_{o, e}=\frac{k_{o, e} w_{0}^{2}}{2}$, and $w_{0}$ means the radius of the beam waist in the $z=0$ plane.

The next step in the model is expanding the complex amplitudes $\Psi_{o, e}$ over all the paraxial beams. For example, the complex amplitudes for the Laguerre-Gaussian beams are as follows:

$$
\begin{aligned}
& \Psi_{o}^{(m, l)}=\frac{(x+i y)^{l}}{\left(w_{0} \sigma_{o}\right)^{l}} L_{m}^{(l)}\left(\frac{r^{2}}{w_{0}^{2} \sigma_{o}}\right) \Psi_{o}, \\
& \Psi_{e}^{(m, l)}=\frac{(x+i y)^{l}}{\left(w_{0} \sigma_{e}\right)^{l}} L_{m}^{(l)}\left(\frac{r^{2}}{w_{0}^{2} \sigma_{e}}\right) \Psi_{e},
\end{aligned}
$$

where $L_{m}^{(l)}(x)$ is the Laguerre polynomial. The only difference with a standard form of the $\Psi_{o}^{(m, l)}$ and $\Psi_{e}^{(m, l)}$ beams is the same magnitude of $\sigma_{o}$ in the denominator of Eq. (2) for the ordinary and extraordinary fundamental Gaussian beams.

Now the matrix representation of propagation of the on-axis beam through the crystal (see Eq. (1)) may be rewritten with the aid of block matrix as

$$
\begin{aligned}
\boldsymbol{\Psi} & =\mathbf{e}_{+} \Psi_{+}(r, z)+\mathbf{e}_{-} \Psi_{-}(r, z) \\
& =\left(\begin{array}{c}
\Psi_{+}^{(o)} \\
\Psi_{-}^{(o)} \\
\Psi_{+}^{(e)} \\
\Psi_{-}^{(e)}
\end{array}\right)=\left(\begin{array}{cccc}
1 & e^{-i 2 \varphi} & 0 & 0 \\
e^{i 2 \varphi} & 1 & 0 & 0 \\
0 & 0 & 1 & -e^{-i 2 \varphi} \\
0 & 0 & -e^{i 2 \varphi} & 1
\end{array}\right)\left(\begin{array}{c}
\Psi_{o}^{(m, l)}(r, z) \\
0 \\
\Psi_{e}^{(m, l)}(r, z) \\
0
\end{array}\right),
\end{aligned}
$$

where $\mathbf{e}_{+}, \mathbf{e}_{-}$are the basis vectors, $\Psi_{+}^{(m, l)}(r, \varphi, z)=a(\varphi) \Psi_{o}^{(m, l)}(r, z)+b(\varphi) \Psi_{e}^{(m, l)}(r, z)$, $\Psi_{-}^{(m, l)}(r, \varphi, z)=c(\varphi) \Psi_{o}^{(m, l)}(r, z)+d(\varphi) \Psi_{e}^{(m, l)}(r, z), \quad$ and the functions 
$a(\varphi), b(\varphi), c(\varphi), d(\varphi)$ are defined by the matrix in Eq. (5). In our model the crystal is described by the block matrix that depends only on geometrical parameters (the azimuth angle $\varphi$ in our case). The material parameters of the crystal (the refractive indices $n_{o}$ and $\left.n_{e}\right)$ are taken into account in the functions of the beams $\Psi_{o}^{(m, l)}$ and $\Psi_{e}^{(m, l)}$, i.e. in the same way as has been done in the simplified model reported in $[11,12]$. However, in our case the beam amplitudes depend on the crystal length.

In order to generalise this model for the tilted beams transmitting at some angle $\alpha$ with respect to the optic axis of crystal, we make use of a standard procedure [6, 17]: if the beam axis is tilted over the angle $\alpha_{o}$ to the optic axis in the plane $z 0 y$, the coordinate $y$ is substituted by $y \rightarrow y+i \alpha_{o} z_{o}$ in both the ordinary and extraordinary amplitudes given by Eqs. (2). Besides, the both amplitudes are multiplied by the factor $f\left(\alpha_{o}\right)=\exp \left(-k_{o} z_{o} \alpha_{o}^{2} / 2\right)$. In frame of our model, the coordinate $y$ in the rest of the functions is substituted according to the following rule: we have $y \rightarrow y-\alpha_{e} z$ for the ordinary beam and $y \rightarrow y-\alpha_{o} z$ for the extraordinary one. Thus, Eqs. (3) and (4) are transformed to

$$
\begin{aligned}
& \Psi_{o}^{(m, l)}=\frac{\left[x+i\left(y-\alpha_{o} z\right)\right]^{l}}{\left(w_{0} \sigma_{o}\right)^{l}} L_{m}^{(l)}\left[\frac{x^{2}+\left(y-\alpha_{o} z\right)^{2}}{w_{0}^{2} \sigma_{o}}\right] \Psi_{o}^{\prime}, \\
& \Psi_{e}^{(m, l)}=\frac{\left[x+i\left(y-\alpha_{e} z\right)\right]^{l}}{\left(w_{0} \sigma_{e}\right)^{l}} L_{m}^{(l)}\left[\frac{x^{2}+\left(y-\alpha_{e} z\right)^{2}}{w_{0}^{2} \sigma_{e}}\right] \Psi_{e}^{\prime},
\end{aligned}
$$

with

$$
\begin{aligned}
& \Psi_{o}^{\prime}=\frac{1}{\sigma_{o}} \exp \left[-\frac{x^{2}+\left(y+i \alpha_{o} z_{o}\right)^{2}}{w_{0}^{2} \sigma_{o}}\right] f\left(\alpha_{0}\right), \\
& \Psi_{e}^{\prime}=\frac{1}{\sigma_{o}} \exp \left[-\frac{x^{2}+\left(y+i \alpha_{o} z_{o}\right)^{2}}{w_{0}^{2} \sigma_{e}}\right] f\left(\alpha_{0}\right) .
\end{aligned}
$$

In the matrix characterising the crystal, we use the substitution $e^{i 2 \varphi} \rightarrow \frac{x+i\left(y+i \alpha_{o} z_{o}\right)}{x-i\left(y+i \alpha_{o} z_{o}\right)}$, so that Eq. (5) would result in

$$
\boldsymbol{\Psi}^{\prime}=\left(\begin{array}{cccc}
1 & \wp_{-} & 0 & 0 \\
\wp_{+} & 1 & 0 & 0 \\
0 & 0 & 1 & -\wp_{-} \\
0 & 0 & -\wp_{+} & 1
\end{array}\right)\left(\begin{array}{c}
\Psi_{o}^{\prime(m, l)} \\
0 \\
\Psi_{e}^{\prime(m, l)} \\
0
\end{array}\right),
$$

where $\wp_{-}=\frac{x-i\left(y+i \alpha_{o} z_{o}\right)}{x+i\left(y+i \alpha_{o} z_{o}\right)}, \quad \wp_{+}=\frac{x+i\left(y+i \alpha_{o} z_{o}\right)}{x-i\left(y+i \alpha_{o} z_{o}\right)} \quad$ and $\quad z_{o} \alpha_{o}=z_{e} \alpha_{e} \quad$ [6]. In this 
representation, the complex amplitude of the beam travelling through the crystal is given by

$$
\begin{aligned}
& \left.\Psi_{+}^{(0,0)}\right|_{\bmod e l}=\Psi_{o}^{(0,0)}+\Psi_{e}^{(0,0)}, \\
& \left.\Psi_{+}^{(0,0)}\right|_{\bmod e l}=\wp_{+}\left(\Psi_{o}^{(0,0)}-\Psi_{e}^{(0,0)}\right),
\end{aligned}
$$

where the incident beam is the right-hand circularly polarised fundamental Gaussian beam at the crystal input $(z=0)$. For comparison, the exact solution of the paraxial wave equation in terms of this study would take the following form [6]:

$$
\begin{gathered}
\left.\Psi_{+}^{(0,0)}\right|_{\text {theory }}=\Psi_{o}^{(0,0)}+\Psi_{e}^{(0,0)} \frac{\sigma_{o}}{\sigma_{e}} \\
\left.\Psi_{-}^{(0,0)}\right|_{\text {theory }}=\wp_{+}\left\{\frac{w_{0}^{2}\left(\Psi_{o}^{(0,0)}-\Psi_{e}^{(0,0)}\right) \sigma_{o}}{x^{2}+\left(x+y+i \alpha_{o} z_{o}\right)^{2}}+\Psi_{o}^{(0,0)}-\Psi_{e}^{(0,0)} \frac{\sigma_{o}}{\sigma_{e}}\right\} .
\end{gathered}
$$

Here we omit the sign "-" in Eq. (12) as a point irrelevant to our model. Generally speaking, the right-hand polarised components $\Psi_{+}^{(m, l)}$ in our model and the theory have much the same form, while the left-hand component given by Eq. (12) includes an additional term. In this respect, our model has little advantage for the case of lowest-order Gaussian beams. On the other hand, high-order fields have a very complex form (see $[6,7]$ ), especially in what concerns the Laguerre-Gaussian and Bessel-Gaussian fields, even for the simplest case of on-axis propagation [18]. Nonetheless, Eqs. (10) hold true for the high-order beams: after substituting $\Psi_{o, e}^{(0,0)} \rightarrow \Psi_{o, e}^{(m, l)}$ we have

$$
\begin{aligned}
& \left.\Psi_{+}^{(m, l)}\right|_{\bmod e l}=\Psi_{o}^{(m, l)}+\Psi_{e}^{(m, l)}, \\
& \left.\Psi_{+}^{(m, l)}\right|_{\bmod e l}=\wp_{+}\left(\Psi_{o}^{(m, l)}-\Psi_{e}^{(m, l)}\right),
\end{aligned}
$$

for different types of paraxial beams. In the latter case, the model suggested here seems to be the only simple way for analytical estimations.

\section{Analysis of the model solutions}

\subsection{Small beam inclinations}

Let us at first compare the intensity profiles derived in frame of the model and the theoretical solutions for the on-axis propagation. The curves typical for the lowest-order Gaussian beam are illustrated in Fig.2a. The model and the theory behave much the same, unless the amplitude of the model solution is slightly larger than that given by the theory. The on-axis Laguerre-Gaussian mode with $m=8, l=0$ (see Fig. 2b) behaves in a similar manner.

As a rule, the most trouble is caused by behaviour of the beam wavefront in the model and theoretical solutions under a small perturbation. In our case, such a perturbation is little deviation of the beam axis from the optic axis of crystal. In 


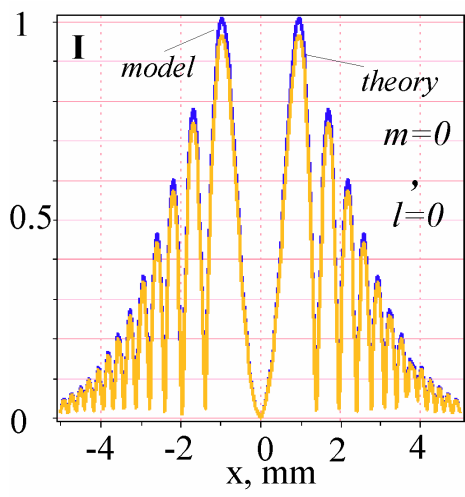

a

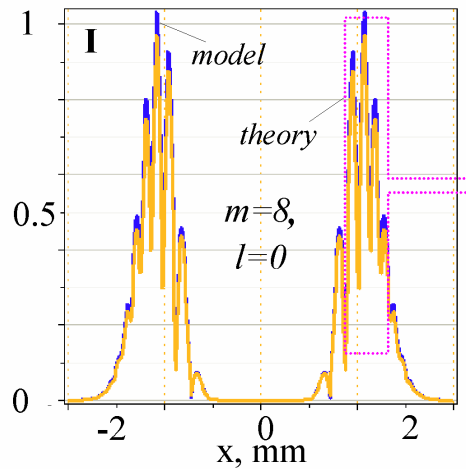

b

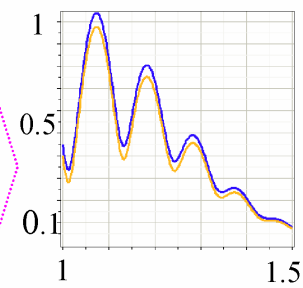

Fig. 2. Comparison of solutions of the model and the theory for the $\Psi$ component of Gaussian ( $m=0, I=0-\mathrm{a})$ and Laguerre-Gaussian $(m=8$, $I=0-b)$ beams with $n_{1}=2.3 ; n_{3}=2.2 ; z=0.4 m, w_{0}=10 \mu \mathrm{m}$ for the case of on-axis propagation $\left(\alpha_{o}=0\right)$.

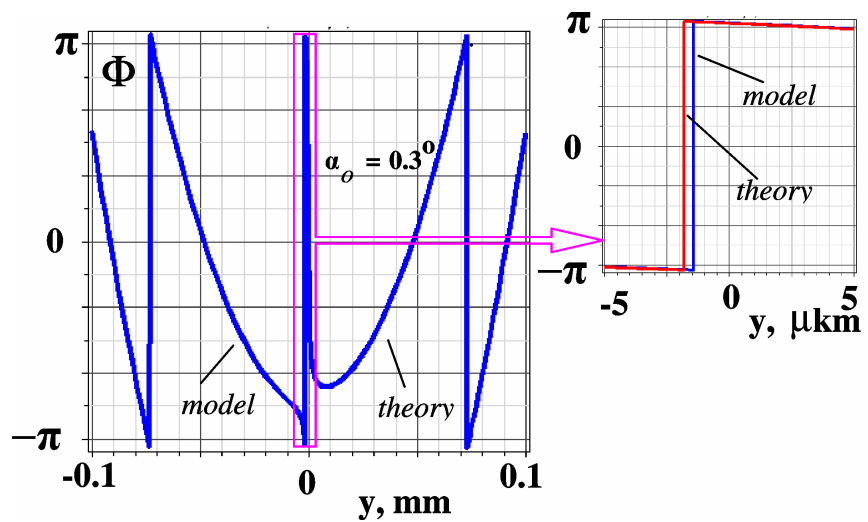

Fig. 3. Phase front destruction in the vicinity of phase singularity in frame of the model and the theory for the lowest-order beam $(m=l=0)$ with $w_{0}=15 \mu \mathrm{m}, z=0.02 \mathrm{~m}$.

framework of the paraxial theory $[4,5]$, the left-hand polarised component gains a centred doubly charged vortex when the initial on-axis beam is right-hand polarised. Fig. 3 illustrates destruction of the wavefront state while tilting the beam axis over the angle $\alpha_{o}$ : the doubly charged vortex splits into two singly charged ones that slide off the beam axis, the model and the theoretical curves coinciding nearly. Little differences in their behaviours refer only to sharp variations of the phase.

\subsection{Vortex trajectories}

When the beam propagates along the optic axis, the vortex topological charge of the lefthand component differs by two units from that of the right-hand component $[4,5]$. The beam inclination turns on mechanisms of interference coupling between the ordinary $\left(\Psi_{o}\right)$ and extraordinary $\left(\Psi_{e}\right)$ beams in each circularly polarised component which, in its 
turn, entails destruction of the high-order optical vortices in the beam components. The individual vortices inside the beam get jumbled. It is difficult to speak what of the beams the optical vortex belongs to. Detailed information about the vortex behaviour in the singular jumble is provided by vortex trajectories plotted in the coordinates ' $(x, y)$ versus $\alpha_{o}^{\prime}: \operatorname{Re}\left\{\Psi_{ \pm}^{(m, l)}\left(x, y, z, \alpha_{o}\right)\right\}=0, \operatorname{Im}\left\{\Psi_{ \pm}^{(m, l)}\left(x, y, z, \alpha_{o}\right)\right\}=0$. Fig. 4 illustrates the vortex trajectories for the simplest case of singly charged initial vortex-beam with the right-hand circular polarisation. The trajectories shown in Fig. 4 are traced by the vortices in the left-hand polarised component of the beam inside the crystal. They are computed for both the theoretical and model solutions. The qualitative portrait of the trajectories in those cases is much the same $[6,7]$. There are three traits in the trajectory behaviour:

The vortex conversion. The initial vortex leaves the beam (see the branch 1 in Fig. 4). Instead, a dipole pair is born near the beam axis, one vortex of which (with the topological charge equal to that of the initial beam) forms the major trunk of the beam core, whereas the second vortex is drawn to the former vortex of the beam sliding off the beam too. Such a vortex conversion results in radical reconstruction of topological structure of the beam. At the same time, the right-hand component lacks this vortex conversion.
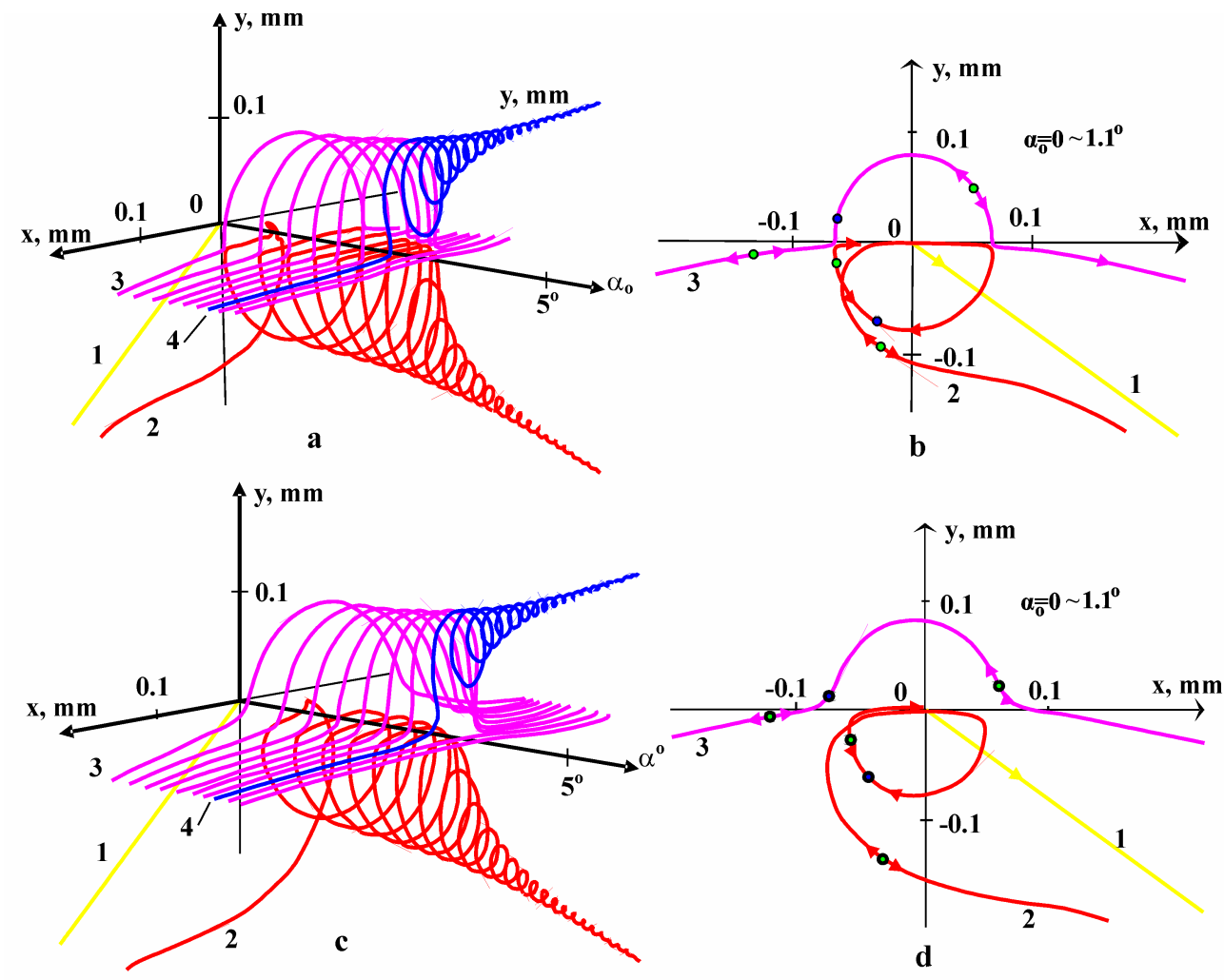

Fig. 4. Vortex trajectories in the model (a, b) and the theory (c. d). 
(1) Shaping of the major trunk. The new-born vortex transmits, together with the tilted beam, near its axis. Two pairs of the vortex dipoles are born again in the vicinity of the axis. One pair of the vortices in the dipole (curve 3) forms new transverse branch of the trajectory (see Fig. 4a, c). The other vortex pair (curve 2) starts to interact with the vortex on the major trunk. As a result, two oppositely charged vortices annihilate, whereas the residual vortex keeps on shaping the major trunk. Note that the vortices on the major trunk and the transverse trajectories do not interact with each other and form two sets of independent trajectories. This shaping process is periodically reproduced as far as to an indistinguishability border $\alpha=\alpha_{\text {border }}$ [13].

(2) The beam splitting. The indistinguishability border is a border for the beam and the crystal parameters to riddle off the nonconforming beams. Not all of the beams can overcome the border but only those corresponding to a characteristic equation [13]. The nonconforming beams keep on propagating without splitting along any crystal length. To shape a splintered beam, one branch of the transverse trajectory is bent (curve 4), twisting around the axis of the second beam crowning the birefringent process. Then the vortices in the splintered beams transmit along screw-like trajectories without dislocation reactions (Fig. 4a, c). This beam splitting is inherent in both the right- and left-hand beam components.

The fine structure of the vortex trajectories is different for the theoretical and model solutions. The projections of the vortex trajectories given by the theory and the model are shown in Fig.4b,d. The transverse and major trajectories approach very close to each other, forming a regular circuit. Only a detailed plotting would allow one to tell them apart. The trajectories of the theory are of strongly selected branches. This, at the first glance, insignificant effect accumulates along the crystal length and contributes essentially to a quadrefringence process in the asymptotic case (to be considered further on).

Simplicity of the presented model enables us to analyse a fine topological structure of the beam travelling through the crystal. The latter is inaccessible for the theoretical solutions because of boundedness of the computation base for the mathematical structures containing numerous singularities. The left-hand component of the birefringent beam just

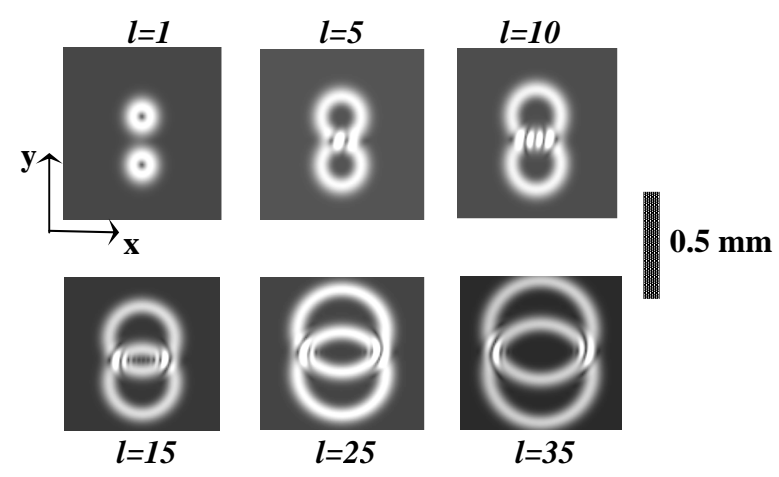

Fig. 5. Splitting of high-order vortex beams with the topological charges / and the parameters $w_{0}=15 \mu \mathrm{m}, z=2 \mathrm{~cm}, n_{o}=2.3, n_{3}=2.2$ and $\alpha_{o}=8^{\circ}$. 


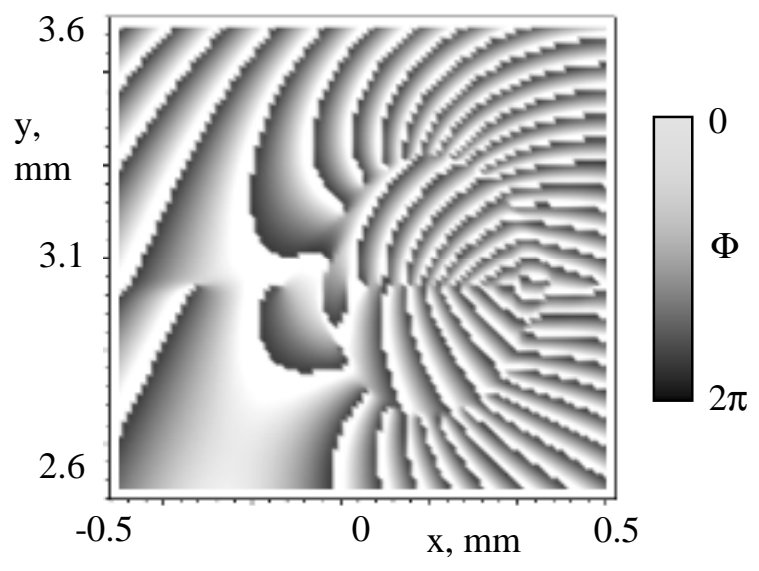

Fig. 6. Phase distribution inside the rings of the $I=25$ vortex-beam composition (see Fig. 5).

represents such an object. Nevertheless, the problem can be easily mastered using the model solution. Indeed, Fig. 5 illustrates the splitting process for the left-hand component of the high-order vortex beams. If the singly charged beam $(l=1)$ with the waist radius $w_{0}=15 \mu \mathrm{m}$ is split at the angle $\alpha_{o}=8^{\circ}$ and the crystal length amounts to $z=2 \mathrm{~cm}$, the beams with $l=5$ are slightly overlapped. The overlapping is aggravated for the beam with $l=8$, whereas the beams with $l=25$ and $l=35$ remain practically indistinguishable. The rings of the beams are overlapped, shaping inside an asymmetric net of optical vortices (see the phase portrait displayed in Fig. 6). The singular net includes not only the dipole pairs but also non-compensated optical vortices. When the beam is tilted, some of the vortices leave the beam axis, forming in the asymptotic case two nearly linearly polarised singular birefringent beams with the identical vortices at the axes.

\subsection{The beam quadrefringence}

The conversion of the initial vortex in the left-hand component distorts phase distribution of the scalar field. Though the initial vortices leave the beam, they remain an ineffaceable trace on the phase portrait of the beam component, which is not obliterated even in the asymptotic case of relatively large angles $\alpha_{o}$ and unboundedly large crystal lengths $z$. This effect can be easily traced in the following way.

The spatial position of the beam is defined by its centre of gravity $x_{C}\left(z, \alpha_{o}\right), y_{C}\left(z, \alpha_{o}\right)$ by means of standard expressions [7, 19]:

$$
\begin{aligned}
& x_{C}=\frac{1}{\mathfrak{I}} \int_{-\infty}^{\infty} d x \int_{-\infty}^{\infty} d x \quad x \quad\left|\mathbf{E}\left(x, y, z, \alpha_{o}\right)\right|^{2}, \\
& y_{C}=\frac{1}{\mathfrak{I}} \int_{-\infty}^{\infty} d x \int_{-\infty}^{\infty} d \quad y \quad y \quad\left|\mathbf{E}\left(x, y, z, \alpha_{o}\right)\right|^{2},
\end{aligned}
$$

where $\mathfrak{I}$ denotes the total beam intensity. Let us consider the asymptotic case when the 
beams are split. Their mutual interference is negligibly small and we can concentrate ourselves only on one individual beam (say, the ordinary one). This beam is centred in the shifted coordinates $\left(x, y^{\prime}=y-\alpha_{o} z\right)$. The factor $\wp_{+}$charged with the inherent topological structure in the left-hand component composes Eq. (14) via Eq. (13) as $\left|\wp_{+}\right|^{2}$. Expanding the function $\left|\wp_{+}\right|^{2}$ into a power series on the parameters of infinitesimality $\left|\frac{x}{\alpha_{o} z}\right|,\left|\frac{y^{\prime}}{\alpha_{o} z}\right|,\left|\frac{z_{o}}{z}\right|<<1, z \rightarrow \infty$ and restricting ourselves only to the linear terms, we arrive at

$$
\frac{\left(x-\alpha_{o} z_{o}\right)^{2}+\left(y^{\prime}+\alpha_{o} z\right)^{2}}{\left(x+\alpha_{o} z_{o}\right)^{2}+\left(y^{\prime}+\alpha_{o} z\right)^{2}}=\frac{\left(x / \alpha_{o} z-z_{o} / z\right)^{2}+\left(y^{\prime} / \alpha_{o} z+1\right)^{2}}{\left(x / \alpha_{o} z+z_{o} / z\right)^{2}+\left(y^{\prime} / \alpha_{o} z+1\right)^{2}} \approx 1-4 \frac{x z_{o}}{\alpha_{o} z^{2}} .
$$

The specific intensity $I_{o,-}$ for the ordinary high-order vortex beam given by Eq. (6) may be rewritten in the polar coordinates $(r, \varphi, z)$ in the form

$$
\begin{aligned}
I_{o,-} & =\left|\Psi_{o,-}^{(l)}\right|^{2}=\left(1-4 \frac{r \cos \varphi z_{o}}{\alpha_{o} z^{2}}\right) \frac{r^{2 l}}{\left(w_{0} \sigma_{o}\right)^{2 l}}\left|\Psi_{o}^{\prime}(r, z)\right|^{2}, \\
r^{2} & =x^{2}+y^{\prime 2} .
\end{aligned}
$$

Then, after simple transformations, we find from Eq. (14) the asymptotic coordinates of the centre of gravity:

$$
\begin{aligned}
& \Delta x_{T}^{(-, o)}=x_{C}^{(-, o)}(z \rightarrow \infty)=-\frac{2}{k_{o} \alpha_{o}}(l+1), \\
& y_{C}^{(-, o)}(z \rightarrow \infty)=0,
\end{aligned}
$$

where we have made use of the approximations $\frac{z^{2}}{z_{o}^{2}} \approx 1+\frac{z^{2}}{z_{o}^{2}}, \frac{z}{z_{o}}>>1$. Similar computation for the right-hand component shows that $x_{C}^{+}(z \rightarrow \infty)=0, \quad y_{C}^{+}(z \rightarrow \infty)=0$. Besides, since $k_{o} \alpha_{o}=k_{e} \alpha_{e}$, the conditions (17) are also true for the left-hand polarised extraordinary beam $\quad\left(\Delta x_{T}^{(-, o)}(z \rightarrow \infty)=\Delta x_{T}^{(-, e)}(z \rightarrow \infty) \quad\right.$ and $\left.y_{C}^{(-, o)}(z \rightarrow \infty)=y_{C}^{(-, e)}(z \rightarrow \infty)\right)$. If we change the initial circular polarisation into its opposite, then the transverse shift $\Delta x_{T}$ reverses its sign, too. The computer simulations of this model process agree well with the above asymptotic result.

Hence, the beams after birefringent crystal are subjected to a transverse shift, the latter being different for the right- and left-hand beam components. In fact, the beam experiences a second splitting. A sketch of this process is shown in Fig. 7. We have earlier called the above phenomenon as a beam quadrefringence [6, 7]. Now we have described it in terms of the matrix model. However, there is some discrepancy between the two approaches to be discussed below. 


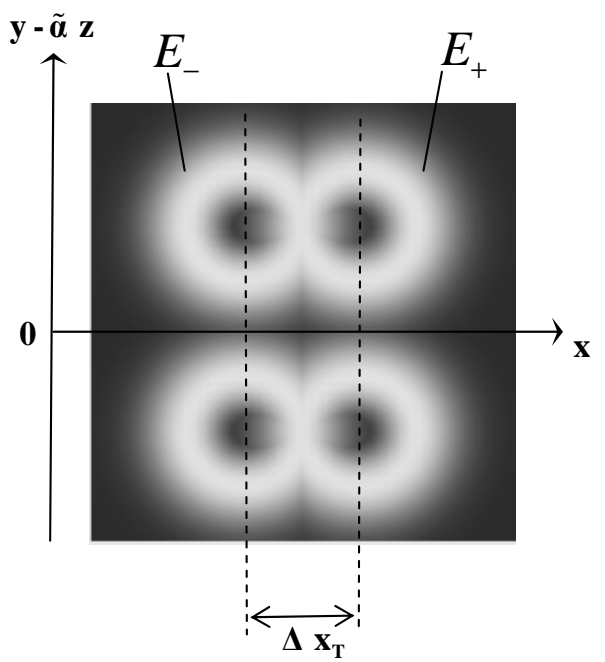

Fig. 7. Sketched representation of vortex beam quadrefringence. The transverse shift $\Delta x_{T}$ is essentially exaggerated $\left(\Delta x_{T} \propto \lambda\right)$ and $\tilde{\alpha}=\left(\alpha_{o}+\alpha_{e}\right) / 2$.

Eq. (17) shows that, apart from the handedness of the initial circular polarisation, the transverse shift $\Delta x_{T}^{(-, o)}$ depends also on the vortex topological charge $l$. At the first sight, it seems that the vortex carries over both the spin $\left(s_{z}\right)$ and orbital $\left(l_{z}\right)$ angular momentum fluxes [20] contributing to the transverse shift $\Delta x_{T}^{(-, o)}$, the orbital angular momentum being proportional to the topological charge $l$ of the vortex. Consequently, the transverse shift should be defined as

$$
\Delta x_{T}^{(e, o)}=-\frac{2}{k_{o} \alpha_{o}}(l+s),
$$

where $s= \pm 1$ is the handedness (or, simply, the spin) of the initial beam.

Let us inspect this assumption on the basis of conservation law for the angular momentum flux [14]. For the centre of gravity of the beam, the conservation law may be written as follows [7, 19]:

$$
\left(\mathbf{r}_{c} \times \mathbf{k}_{c}\right)_{z}+\left(\mathfrak{I}_{+}-\mathfrak{I}_{-}+\mathfrak{I}_{+} l_{+}+\mathfrak{I}_{-} l_{-}\right) \frac{k_{z}}{k}=\text { const },
$$

where $\mathbf{r}_{c}$ is the radius vector of the centre of gravity, $\mathbf{k}_{c}$ and $k_{z}$ stand respectively for the wave vector and its $z$-component associated with the centre of gravity, $l_{+}$and $l_{-}$the vortex topological charges respectively for the right- and left-hand components, and $\mathfrak{I}_{ \pm}$ the normalised intensities of the beam components. Since the inclination angle is small, we can assume that $k_{z} / k \approx 1$. In the $z=0$ plane, the terms in Eq. (18) referred to our vortex-beam may be defined as $\mathfrak{I}_{+}(z=0)=1, \mathfrak{I}_{-}(z=0)=0$ and $\left.\left(\mathbf{r}_{c} \times \mathbf{k}_{c}\right)\right|_{z=0}=0$. The vortex conversion (see Sec. III) results in identity of the topological vortex charges in the 
asymptotic case $l_{+}=l_{-}, z \rightarrow \infty$. Besides, the beam is depolarised $\left(\mathfrak{I}_{+}(z \rightarrow \infty)=\mathfrak{I}_{-}(z \rightarrow \infty)\right)$, so that, ultimately, we have to rewrite Eq. (18) for the general case of the initial beam with the handedness $s: l+s=l+\left(\mathbf{r}_{c} \times \mathbf{k}_{c}\right)_{z}$. At the point of origin of the coordinate system given in Fig. 1 the vector product is equal to $\left.\left(\mathbf{r}_{c} \times \mathbf{k}_{c}\right)_{z}\right|_{z \rightarrow \infty}=-k_{o} \alpha_{o} \Delta x_{T}$, from whence we find

$$
\Delta x_{T}=-\frac{s}{k_{o} \alpha_{o}} .
$$

Since the transverse shift of the right-hand component is zero $\left(\Delta x_{T}^{(+)}=0\right)$, the lateral displacement of the left-hand component $\Delta x_{T}^{(-)}$amounts to doubled $\Delta x_{T}: \Delta x_{T}^{(-)}=2 \Delta x_{T}$. Eq. (19) indicates that the lateral shift is proportional to the wavelength $\lambda$ and inversely proportional to the inclination angle $\alpha_{o}$ of the centre of gravity.

Thus, the transverse shift of the beams represents a consequence of the conservation law for the angular momentum flux. The transverse shift depends only on the handedness of circular polarisation of the initial beam, whereas the model solution gives somewhat overrated magnitude for high-order beams with $l \neq 0$. Such mismatch of the asymptotics is apparently caused by the fact that the model employs the beams in the left-hand component having the Gaussian envelope, while the beams obtained theoretically have more complex envelope functions.

\section{Conclusions}

We have considered a matrix model for the propagation of tilted vortex beam in an optically uniaxial crystal and have compared the results thus obtained with the solutions of the paraxial wave equation. The model deals with the propagation of two circularly polarised beams. One beam, whose circular polarisation is identical to that of the initial beam, consists of two co-phased vortex beams with the Gaussian envelope, propagating at some angle to each other. The second, orthogonally polarised beam includes two antiphased beams, with an additional phase factor.

Contrary to the models suggested earlier, our matrix model predicts the vortex conversion, the traits of the vortex trajectories and the beam quadrefringence. It is in a good agreement with the paraxial theory. Besides, the matrix model has enabled us to calculate the complex vortex behaviour of high-order singular beams in a much simpler way when compare with the paraxial theory. However, the model imposes overrated magnitudes for the high-order beam parameters in the asymptotic case. This circumstance has to be taken into account when estimating vortex transformations in complex singular processes.

\section{Acknowledgement}

One of us (A. V. V.) thanks Prof. Vlokh R. for a series of important remarks that stimulated the present study.

Ukr. J. Phys. Opt. 2009, V10, №3 


\section{References}

1. Soskin M and Vasnetsov M, 2001. Singular optics. Progress in Optics 42: 219-276.

2. Fleck J A and Feit M D, 1983. Beam propagation in uniaxial anisotropic media. J. Opt. Soc. Amer. 73: 920-928.

3. Seshadri S R, 2003. Basic elliptical Gaussian wave and beam in a uniaxial crystal. J. Opt. Soc. Amer. A. 20: 1818-1826.

4. Volyar A and Fadeyeva T, 2003. Generation of singular beams in uniaxial crystals, Opt. Spectr. 94: 264-274.

5. Ciattoni A, Cincotti G and Palma C, 2003. Circular polarized beams and vortex generation in uniaxial media. J. Opt. Soc. Amer. A. 20: 163-171.

6. Fadeyeva T, Rubass A, Egorov Yu, Volyar A and Swartzlander G, 2008. Quadrefringence of optical vortices in a uniaxial crystal. J. Opt. Soc. Amer. A. 25: 1643-1641.

7. Fadeyeva T, Rubass A and Volyar A, 2009. The transverse shift of a high order paraxial vortex-beam induced by a homogeneous anisotropic medium. Phys. Rev. A. 79: 053815-1-12. See also ArXiv http://arxiv.org/abs/0812.3498

8. Brasselet E, Izdebskaya Ya, Shvedov V, Desyatnikov A., Krolikowsky W and Kivshar Yu, 2009. Dynamics of optical spin-orbit coupling in uniaxial crystals. Opt. Lett. 34: 1021-1023.

9. Volyar A and Fadeyeva T, 2006. Laguerre-Gaussian beams with complex and real arguments in uniaxial crystals. Opt. Spectr. 101: 297-304.

10. Volyar A, Rubass A, Shvedov V, Fadeyeva T and Kotlyarov K, 2007. Optical vortices and Airy' spiral in chiral crystals. Ukr. J. Phys. Opt. 8: 166-181.

11. Flossmann F, Schwarz U T, Maier M and Dennis M R, 2005. Polarization singularities from unfolding an optical vortex through a birefringent crystal. Phys. Rev. Lett. 95: 253901-4.

12. Flossmann F, Schwarz U T, Maier M and Dennis M R, 2006. Stokes parameters in the unfolding of an optical vortex through a birefringent crystal. Opt. Expr. 14: 11402-11411.

13. Fadeyeva T, Egorov Yu, Rubass A, Swartzlander G A, Jr and Volyar A, 2007. Indistinguishability limit for off-axis vortex beams in uniaxial crystals. Opt. Lett. 32: 3116-3118.

14. Ciattoni A, Cincotti G and Palma C, 2003. Angular momentum dynamics of a paraxial beam in uniaxial crystal. Phys. Rev. E. 67: 036618-1-10.

15. Azzam R M A and Bashara N M. Ellipsometry and polarized light (North-Holland, 1977).

16. Born M and Wolf E. Principles of optics. New York: Cambridge University Press (1999).

17. Chin S Y and Felson L B, 1974. Gaussian beam in anisotropic media. Appl. Phys. 5: 225-239. 
18. Cincotti G, Ciattoni A and Palma C, 2002. Laguerre-Gauss and Bessel-Gauss beams in uniaxial crystals. J. Opt. Soc Am. A. 19: 1680-1688.

19. Bliokh K Yu and Bliokh $\mathrm{Yu} \mathrm{P,} \mathrm{2007.} \mathrm{Polarization,} \mathrm{transverse} \mathrm{shifts,} \mathrm{and} \mathrm{angular}$ momentum conservation laws in partial reflection and refraction of an electromagnetic wave packet. Phys. Rev. E. 75: 066609-1-10.

20. Allen L, Barnet S M and Padgett M J. Optical Angular Momentum Bristol: IOP Publishing (2003).

Fadeyeva T.A., Rubass A.F. and Volyar A.V., 2009. The matrix model of vortex-beam quadrefringence in a uniaxial crystal. Ukr.J.Phys.Opt. 10: 109-123.

Анотація. В роботі розглянута матрична модель для сингулярних променів, які поширюються під певним кутом до оптичної осі в оптично одновісних кристалах. При узгодженні з параксіальною теорією, модель передбачає серію тонких ефектів таких, як конверсія вихорів та чотиризаломлення променів. Крім иього на основі даної моделі отримуються параметри променя в асимптотичному випадку. Це дає можливість розрахувати прочеси, які виникають у променях, що містять вихори вищих порядків і для яких існуюча теорія зіштовхується з розрахунковими труднощами. 